\title{
APPLICATION OF MULTI-VALUED WEIGHTING LOGICAL FUNCTIONS IN THE ANALYSIS OF A DEGREE OF IMPORTANCE OF CONSTRUCTION PARAMETERS ON THE EXAMPLE OF HYDRAULIC VALVES
}

\author{
A. DEPTUŁA \\ Opole University of Technology \\ Faculty of Production Engineering and Logistic \\ 75 Ozimska Str., 45-370 Opole, POLAND \\ E-mail: a.deptula@po.opole.pl
}

\begin{abstract}
In the optimization process, changes in the construction parameters value influence the behaviour of functions depending on time. Weighting logical coefficients for the stabilisation time are taken into consideration here, i.e., a shorter (better) stabilisation time has a more important (bigger) value of the weighting coefficient. An example of applying weighting logical functions in the analysis of a degree of importance of construction parameters of a hydraulic valve is presented in the paper.
\end{abstract}

Key words: optimization process, weighting logical coefficients, hydraulic valves, pomps.

\section{Introduction}

Among tools supporting decision making processes, it is possible to differentiate decision tables and trees, dendrites, tree classifiers as well as graphs. These tools are included in the so-called decision support methods based on graphs. A set of decisions (and relations between them) is written in a graphic way out of a mathematical model which is the main basis of the decision process realisation which a decision-maker can use in order to solve problems of any kind. Modelling of the whole process is necessary in the case of supporting decision-making processes in the optimization of mechanical systems. A lot of different feedbacks between elements of internal structure can be differentiated in the objects being analysed (e.g., mechanical systems). It is necessary to use an appropriate graphic model where the cause and effect relationship, which takes place inside an analysed object, occurs. Fluid-flow machines form a vast group of sets used in industry (Francis and Betts, 1997; Giergiel, 1990; Kurowski, 2001). Decision tables and logical functions (Deptuła, 2014; Stępniewski, 1994) can be applied in the issues of modelling machine systems with differential equations (ordinary and partial ones). It results from the fact that non-linear elements can be divided into a finite number of linear elements (parts) what leads to getting several linear systems. Discrete optimization of fluid-flow machines (Suzuki and Urata, 2003; Żak and Stefanowski, 1994) is based on indicating the degree of importance of construction and exploitation parameters. Guidelines concerning the sequence of making decisions result from multi-valued decision trees and taking into consideration the realisation of the assumed purpose function (e.g., the system stabilisation).

\section{Multi-valued logical functions with weighting coefficients}

Graphs of output data with the indicated stabilisation time as modelling results (e.g., in the programs such as: Matlab, Fluent ) depend on given data of construction parameters. Changes in such values (e.g., decreasing, increasing, remaining unchanged), in the process of designing the set for different work 
conditions can be written in the form of a code in multi-valued logic, whereas the set of design guidelines can be presented as a sum of multi-valued logical products.

\subsection{Weighting coefficients}

In the partial multi-valued logical function of $n$ variables $\left(m_{1}, \ldots, m_{n}\right)$ - valued, the weighting coefficient $w_{i}$ before the canonical product has the value within this scope $\left\langle w_{1}, \ldots, \mathrm{w}_{n}\right\rangle$, if $w_{j}=w_{j-1}+w_{j-2}+\ldots+w_{1}$, where $j=2, \ldots, n$. Therefore, function weights in the graph in Fig. 1 can be described by the following set of logical equations: $f_{2}: w_{2}(0,1,2)$, which means that the $f_{0}$ stabilisation is obtained the fastest and the $f_{2}$ stabilisation is obtained the latest, which means $w_{0}>w_{1}>w_{2}$ (Deptuła, 2011; Deptuła and Partyka, 2010), that is $f_{i}$ and $f_{j}, w_{i}<w_{j}$, if $t_{t}>t_{j}$.

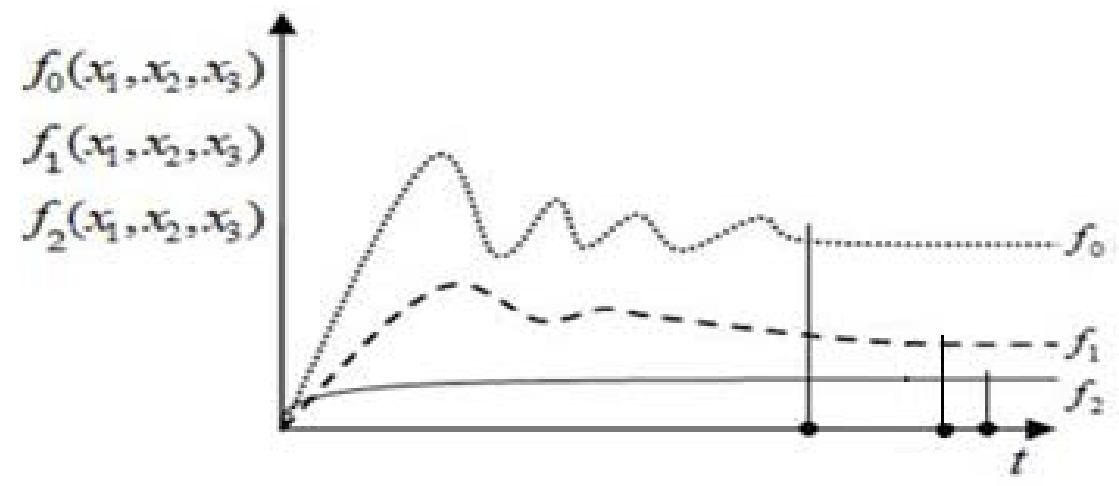

Fig.1. Graphs of the functions $f_{0}, f_{1}, f_{2}$ depending on time for the coded version of multi-valued decision variables $x_{1}=0, x_{2}=1, x_{3}=2$.

Then, an alternative, multi-valued normal form is created where a bigger logical weighting coefficient means a shorter stabilisation time (Deptuła and Partyka, 2012).

It is possible to apply the Quine - McCluskey algorithm of multi-valued functions minimization in multi-valued logical functions with weighting products (Deptuła, 2014).

\section{Sets of multi-valued logical equations with weighting products}

In the case of flow rate calculations (e.g., in positive-displacement pumps or centrifugal pumps) many characteristics are taken into account at the same time and they form the set of functions $f_{1}, f_{2}, f_{3}, \ldots, f_{n}$.

The set of equations can be defined as $Y_{S}^{R}$

$$
Y_{S}^{R}:\left\{\begin{array}{l}
R_{i}=r_{i}+r_{i+j}+\ldots+r_{n}, \\
R_{i+1}=r_{i}+r_{i+j}+\ldots+r_{n}, \\
R_{m}=r_{i}+r_{i+j}+\ldots+r_{n} .
\end{array}\right.
$$


where: $R$ - the set of logical equations- $R:\left\{R_{i}, R_{i+1}, \ldots, R_{m}\right\}, S$ - the set of canonical products as elements$\mathrm{S}:\left\{r_{i}, r_{i+1}, \ldots, r_{n}\right\}$, if: $S \subset R, R_{i}=r_{i}+r_{i+1}+, \ldots,+r_{n}$.

The set of multi-valued logical equations is presented in the form of a morphological table where the number of verses is equal to the number of equations $R_{j}, \boldsymbol{j}=1, \ldots, m$.

The set of multi-valued logical equations can be solved using combinatorics in view of morphological analysis with maintaining postulates of the Rosser-Turguette system (Deptuła, 2014; 2011).

\section{Example 1.}

Logically presented theoretical possibilities of changes in the numbers of construction parameters have the following form: $x_{1}=0,1, x_{2}=0,1,2, x_{3}=0,1$, (where the sign " " means "to be kept unchanged" and it was obtained out of modelling of the set of two multi-valued logical equations for output data (Deptuła and Partyka, 2012)

$$
\left\{\begin{array}{l}
y_{1}(t)=1(001)+1(011)+2(021)+1(000), \\
y_{2}(t)=1(001)+2(011)+3(021)+1(000),
\end{array}\right.
$$

After minimization, the real solution can be written in the following form

$$
y=1 \cdot 001+1 \cdot 011+2 \cdot 021+1 \cdot 000, \quad \text { what results in: } 1 \cdot(00-)+1 \cdot(0-1)+2 \cdot(021) .
$$

\section{Weighting multi-valued logical functions in the analysis of a degree of importance of construction parameters of the overflow valve}

The overflow valve is applied in the systems in order to let the excess of fluid flow to container where the pump efficacy is bigger than the need. An example of a drive system of an actuator with an overflow valve is presented in Fig.2 (Deptuła, 2014; Żak and Stefanowski, 1994; Smith, 2003).

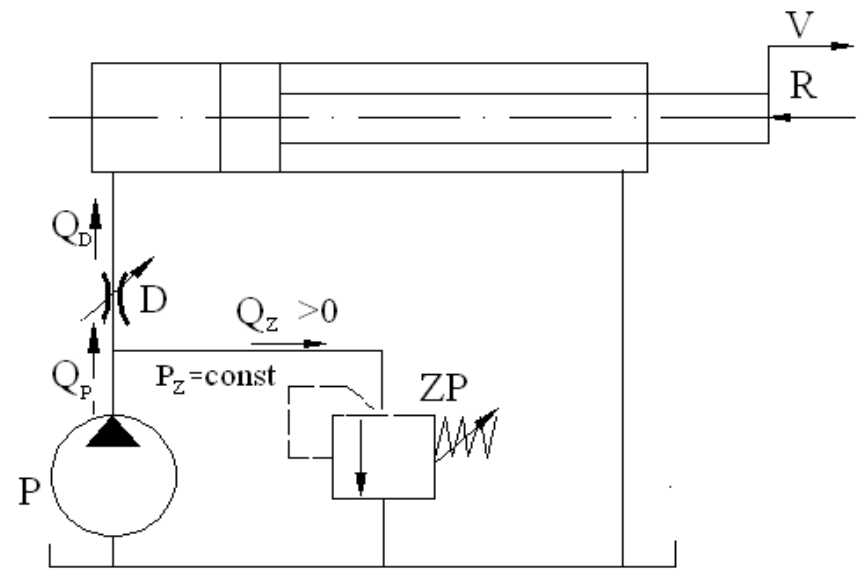




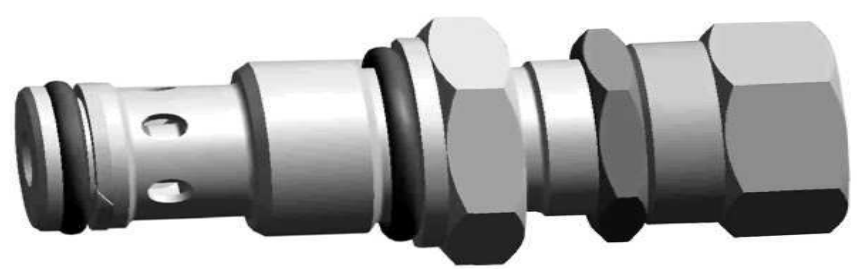

Fig.2. A scheme of an actuator with an overflow valve.

The equation of forces acting on the closing component of a valve is presented in the following way (Deptuła, 2014)

$$
\frac{Q_{p}{ }^{2}}{A_{l}} \rho+\rho \cdot A_{2}+\rho \cdot l \frac{d Q_{p}}{d t}=G_{a p}+S+k \cdot x+f \frac{d x}{d t}+m \frac{d^{2} x}{d t^{2}}+\Phi \sqrt{2 \cdot \rho} \cdot \cos (v) \cdot Q_{p} \sqrt{p},
$$

whereas equations of flows have the following form

$$
\begin{aligned}
& Q=\mu \cdot K \cdot x \sqrt{p}+A_{1} \frac{d x}{d t}+\frac{V}{B} \frac{d p}{d t}, \\
& Q_{p}=\mu \cdot K \cdot x \sqrt{p}+A_{l} \frac{d x}{d t}
\end{aligned}
$$

where

$$
K=\pi \cdot d_{m} \sqrt{\frac{2}{\rho}} .
$$

Equations of the valve work in a dimensionless form used to make simulation are presented in the following form

$$
\begin{aligned}
& \rho \frac{Q_{o}{ }^{2}}{A_{1} S_{o}} Q_{p w}^{2}+\frac{A_{2} p_{o}}{S_{o}} p_{w}+\frac{T_{Q p}}{T_{o}} \frac{d Q_{p w}}{d t_{w}}=1+\frac{k x_{o}}{S_{o}} x_{w}+\frac{T_{f}}{T_{o}} \frac{d x}{d t_{w}}+\left(\frac{T_{m s}}{T_{o}}\right)^{2} \frac{d^{2} x}{d t_{w}^{2}}+ \\
& +\Phi \frac{\sqrt{2 \rho}}{S_{o}} \cos (v) Q_{o} Q_{p w} \sqrt{p_{o}} \sqrt{p_{w}}, \\
& Q_{w}=\mu x \sqrt{p_{w}}+\frac{T_{A}}{T_{o}} \frac{d x}{d t_{w}}+\frac{d p_{w}}{d t_{w}}, \\
& Q_{p w}=\mu x \sqrt{p_{w}}+\frac{T_{A}}{T_{o}} \frac{d x}{d t_{w}} .
\end{aligned}
$$




\subsection{Weighting coefficients}

In order to make a discrete optimization, changes in parameters have been coded in the following way: 0- large decrease, 1- small decrease, 2- without changes, 3-increase, 4- large increase (for $m$ and $k$ ) and : 0- small decrease, 1- without changes, 2- increase (for $d$ ). For example, a combination of changes 122 means a small decrease in mass $m$, leaving the spring constant without changes $k$ and an increase of the diameter $d$. On the other hand, the combination $\mathbf{4 0 2}$ means a large increase in mass $m$, a large decrease of the spring constant $k$ and an increase of the diameter $d$ in relation to the adopted arithmetic values in the early stage of designing. Depending on the adopted combinations of code changes in parameters $m, k$ and $d$ in canonical products, the behaviour of functions which depend on time is different.

When we look at the behaviour of the functions $x, Q$ and $p$ at the time of stabilization $t_{w}<200 t_{o}$ (strict condition), if there are products of code changes of parameters $m, k$ and $d$ the following values of weighting parameters have been adopted:

$$
-w_{i}=4, t_{w} \leq 50 t_{o} ; w_{i}=3,50 t_{o}<t_{w} \leq 100 t_{o} ; w_{i}=2,100 t_{o}<t_{w} \leq 150 t_{o} ; w_{i}=1,150 t_{o}<t_{w} \leq 200 t_{o} .
$$

When we increase the stabilisation time to $t_{w}<800 t_{o}$ (liberal work conditions), it has been assumed that:

$$
-w_{i}=4, t_{w} \leq 200 t_{o} ; w_{i}=3,200 t_{o}<t_{w} \leq 400 t_{o} ; w_{i}=2,400 t_{o}<t_{w} \leq 600 t_{o} ; w_{i}=1,600 t_{o}<t_{w} \leq 800 t_{o} .
$$

The weighting coefficient $w_{i}$ in the case of code indications depends on the stabilisation time $t$, but $l_{i}<l_{j}$ if $t_{i}>t_{j}$. Figure 3 shows exemplary plots of functions $x, Q$ and $p$, when appropriate changes of code combinations $m, k$ and $d$ occur. Appropriate products of combinations in code changes have the following weighting coefficients:

- in the case of a limitation $t_{w}<200 t_{o}: x: 2 \cdot(222)+1 \cdot(212)+1 \cdot(121) ; Q: 2 \cdot(222)+3 \cdot(212)$; $p: 2 \cdot(222)+2 \cdot(212)$

- in the case of a limitation $t_{w}<800 t_{o}: \quad x: 4 \cdot(222)+4 \cdot(212)+4 \cdot(121) ; \quad Q$ : $4 \cdot(222)+4 \cdot(212)+3 \cdot(212) ; p: 4 \cdot(222)+4 \cdot(212)+3 \cdot(212)$.

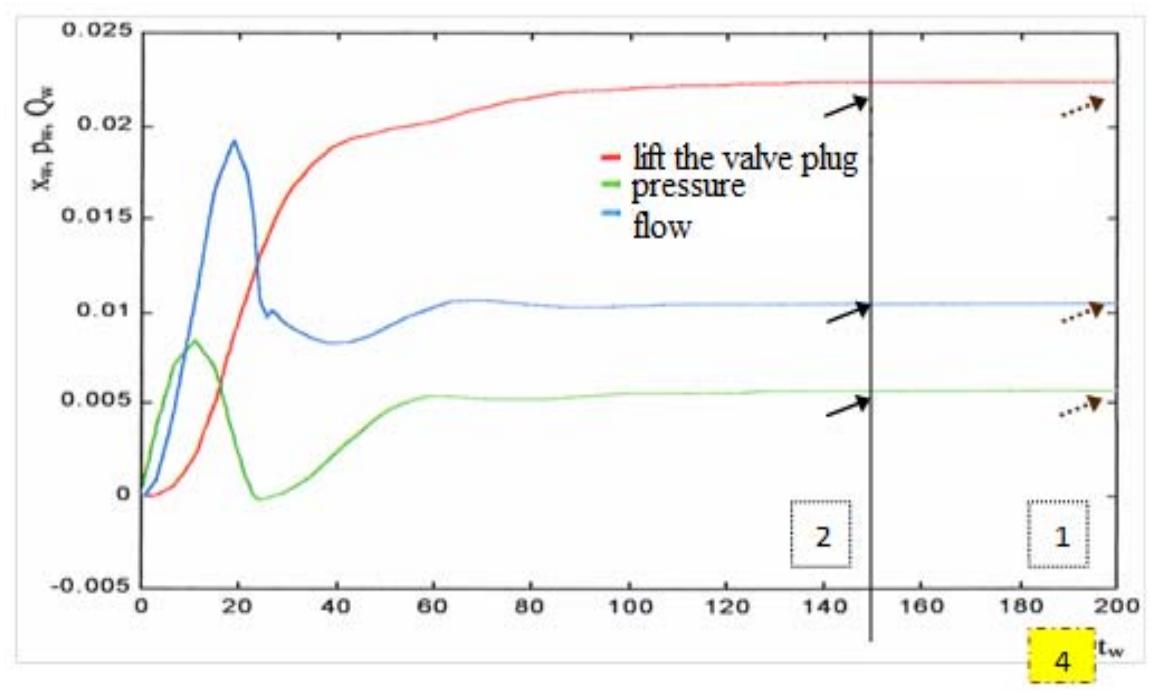



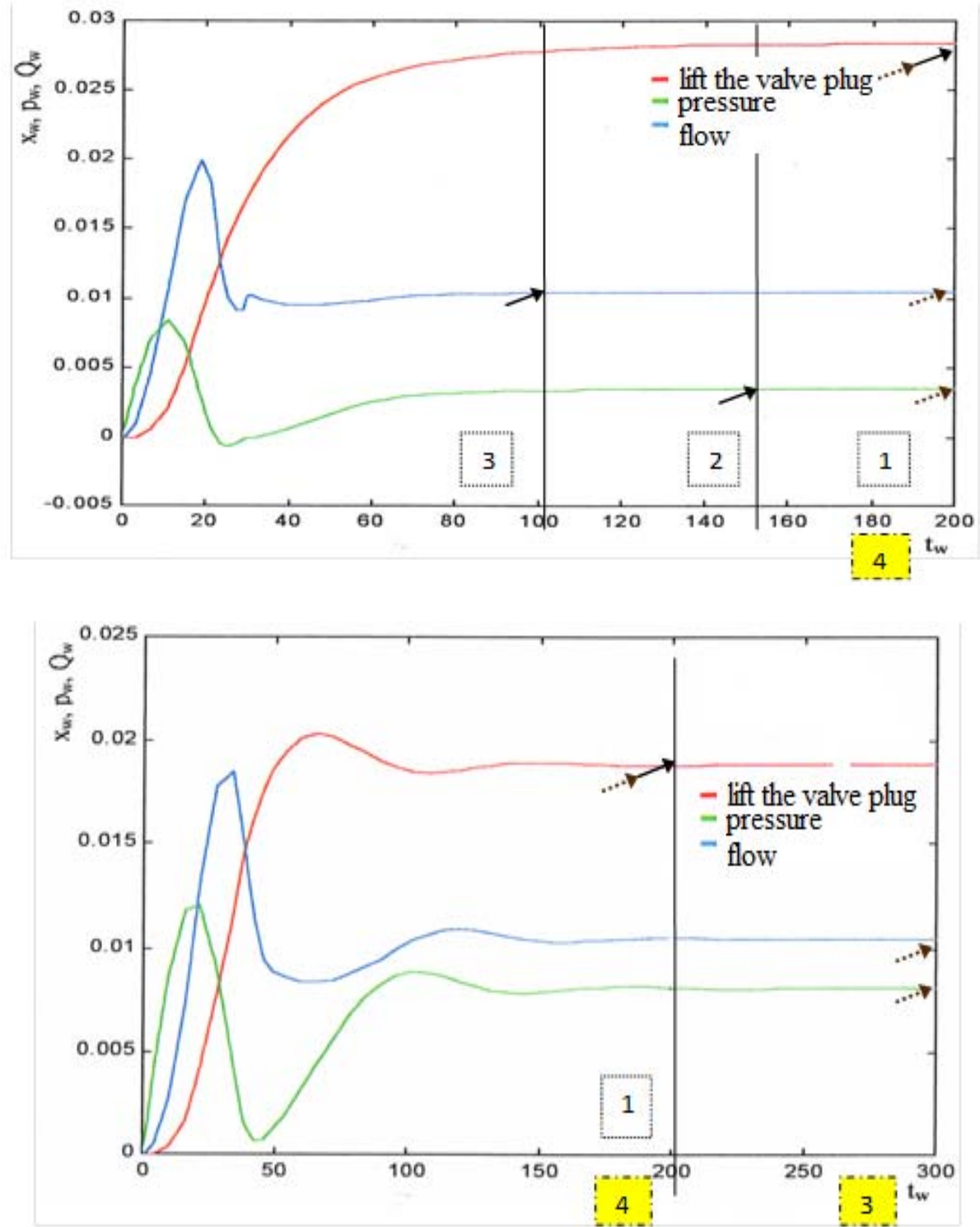

Fig.3. Time function plots $x, Q, p$ with the indicated stabilisation time and weighting coefficients in the case of limitations: $t_{w}<200 t_{o} \quad \rightarrow$ and $t_{w}<800 t_{o}-\cdots \rightarrow$ of code changes in parameters: $m, k$ and $d: 222 ; 212,121$ (Deptuła, 2014).

Table 1. KAPN of changes in parameter values $m, k$ and $d\left(t_{w}<200 t_{o,} \frac{w_{\max }}{w_{\text {stab. }}}<3.6\right)$.

\begin{tabular}{|c|c|c|c|c|c|c|c|c|}
\hline$m$ & $k$ & $d$ & $m$ & $k$ & $d$ & $m$ & $k$ & $d$ \\
\hline 2 & 2 & 2 & 1 & 0 & 2 & 0 & 1 & 2 \\
\hline 2 & 1 & 2 & 0 & 2 & 1 & 0 & 0 & 1 \\
\hline 1 & 2 & 1 & 0 & 2 & 2 & 0 & 0 & 2 \\
\hline 1 & 2 & 2 & 0 & 1 & 1 & 0 & 3 & 2 \\
\hline 1 & 1 & 1 & 1 & 1 & 2 & 1 & 3 & 2 \\
\hline 2 & 0 & 2 & \multicolumn{10}{|l}{}
\end{tabular}


In the case of the overflow valve, a set of three multi-valued logical equations of output data $x, Q, p$ was obtained out of modelling (Deptuła, 2014) respectively for a limiting condition

a) $t_{w}<200 t_{o}$

$$
Y_{t_{w}<200 t_{o}}=\left\{\begin{array}{l}
x=2 \cdot 222+1 \cdot 212+1 \cdot 121+2 \cdot 122+1 \cdot 111+2 \cdot 112+1 \cdot 132+2 \cdot 202+2 \cdot 102+ \\
+2 \cdot 021+3 \cdot 022+3 \cdot 032+2 \cdot 011+3 \cdot 012+1 \cdot 001+3 \cdot 002 \\
Q=2 \cdot 222+3 \cdot 212+3 \cdot 122+1 \cdot 111+4 \cdot 112+3 \cdot 132+3 \cdot 202+4 \cdot 102+3 \cdot 021+4 \cdot 022+ \\
+3 \cdot 032+4 \cdot 011+4 \cdot 012+4 \cdot 001+4 \cdot 002+2 \cdot 312+3 \cdot 300+3 \cdot 101+2 \cdot 000+2 \cdot 100 \\
p=2 \cdot 222+2 \cdot 212+3 \cdot 122+1 \cdot 111+3 \cdot 112+2 \cdot 132+2 \cdot 202+3 \cdot 102+ \\
+2 \cdot 021+3 \cdot 022+3 \cdot 032+2 \cdot 011+3 \cdot 012+1 \cdot 001+3 \cdot 002+1 \cdot 101
\end{array}\right.
$$

b) $t_{w}<800 t_{o}$

$$
Y_{t_{w}<800 t_{o}}\left\{\begin{array}{l}
x=4 \cdot 222+4 \cdot 212+4 \cdot 121+4 \cdot 122+4 \cdot 111+4 \cdot 112+4 \cdot 132+ \\
+4 \cdot 202+4 \cdot 102+4 \cdot 021+4 \cdot 022+4 \cdot 032+4 \cdot 011+4 \cdot 012+4 \cdot 001+ \\
+4 \cdot 002+3 \cdot 322+3 \cdot 312+3 \cdot 031+3 \cdot 010+3 \cdot 232+3 \cdot 300+3 \cdot 020+ \\
+2 \cdot 221+2 \cdot 332+1 \cdot 211+1 \cdot 201+3 \cdot 101+1 \cdot 422+3 \cdot 000 \\
\\
+4=4 \cdot 222+4 \cdot 212+3 \cdot 121+4 \cdot 122+4 \cdot 111+4 \cdot 112+4 \cdot 132+ \\
+4 \cdot 002+3 \cdot 322+3 \cdot 312+3 \cdot 031+3 \cdot 010+3 \cdot 232+3 \cdot 300+3 \cdot 020+ \\
+2 \cdot 221+2 \cdot 332+1 \cdot 211+1 \cdot 201+4 \cdot 101+1 \cdot 422+3 \cdot 000+1 \cdot 402 \\
\\
p=4 \cdot 222+4 \cdot 212+3 \cdot 121+4 \cdot 122+4 \cdot 111+4 \cdot 112+4 \cdot 132+ \\
+4 \cdot 202+4 \cdot 102+4 \cdot 021+4 \cdot 022+4 \cdot 032+4 \cdot 011+4 \cdot 012+4 \cdot 001+ \\
+4 \cdot 002+3 \cdot 322+4 \cdot 312+3 \cdot 031+3 \cdot 010+3 \cdot 232+4 \cdot 300+3 \cdot 020+ \\
+2 \cdot 221+2 \cdot 332+2 \cdot 211+2 \cdot 201+4 \cdot 101+1 \cdot 422+1 \cdot 412+4 \cdot 000+1 \cdot 100+3 \cdot 402
\end{array}\right.
$$

A solution of the set of equations $Y_{t_{w}<20 t_{\circ}}$ is $5120(16(x) 20(Q) 16(p))$ of theoretical versions of solutions. A solution true for the set $Y_{t_{w}<20 t_{o}}$ obtains the following form

$$
\begin{aligned}
& f(x, Q, p)^{t_{w}<200 t_{o}}=2 \cdot 222+1 \cdot 212+2 \cdot 122+1 \cdot 111+2 \cdot 112+1 \cdot 132+2 \cdot 202+ \\
& +2 \cdot 102+2 \cdot 021+3 \cdot 022+3 \cdot 032+2 \cdot 011+3 \cdot 012+1 \cdot 001+3 \cdot 002= \\
& =1 \cdot(001)+3 \cdot(0-2)+2 \cdot(011)+2 \cdot(021)+1 \cdot(1-2)+2 \cdot(122)+ \\
& +2 \cdot(112)++2 \cdot(102)+(1 \cdot 111)+2 \cdot(202)+1 \cdot(212)+2 \cdot(222) .
\end{aligned}
$$

In the case of the limiting condition $t_{w}<800 t_{o}$, there are $30690(30(x) 33(Q) 31(p))$ real versions of solutions. Solutions can be obtained in the following form 


$$
\begin{aligned}
& f(x, Q, p)^{t_{w}<800 t_{o}}=4 \cdot 222+4 \cdot 212+3 \cdot 121+4 \cdot 122+4 \cdot 111+4 \cdot 112+4 \cdot 132+ \\
& +4 \cdot 202+4 \cdot 102+4 \cdot 021+4 \cdot 022+4 \cdot 032+4 \cdot 011+4 \cdot 012+ \\
& +4 \cdot 001+4 \cdot 002+3 \cdot 322+3 \cdot 312+3 \cdot 031+3 \cdot 010+3 \cdot 232+3 \cdot 300+ \\
& +3 \cdot 020+2 \cdot 221+2 \cdot 332+1 \cdot 211+1 \cdot 201+3 \cdot 101+1 \cdot 422+3 \cdot 000= \\
& =3(00-)+4(001)+4(002)+3(01-)+4(011)+4(012)+3(02-)+ \\
& +4(021)+4(022)+3(0-1)+4(001)+4(011)+4(021)+4(0-2)+ \\
& +4(1-2)+1(-22)+3(322)+4(222)+4(122)+4(022)+4(032)+ \\
& +3(101)+4(111)+3(121)+1(201)+1(211)+4(212)+2(221)+ \\
& +3(232)+3(300)+3(312)+2(332) .
\end{aligned}
$$

If we adopted a very strict condition in the graph of functions $x, Q$ and $p$ : stabilisation time $t_{w}<100 t_{o}$ and weighting coefficients values: $w_{i}=4, t_{w} \leq 25 t_{o} ; w_{i}=3,25 t_{o}<t_{w} \leq 50 t_{o} ; w_{i}=2$, $50 t_{o}<t_{w} \leq 75 t_{o} ; w_{i}=1,75 t_{o}<t_{w} \leq 100 t_{o}$, then a weighting multi-valued set of equations for $x, Q$ and $p$ would have the following form

$$
Y_{t_{w}<100 t_{o}}=\left\{\begin{aligned}
& x=1 \cdot 022+1 \cdot 032+1 \cdot 012+1 \cdot 002 \\
& Q= 1 \cdot 222+1 \cdot 212+1 \cdot 122+3 \cdot 112+2 \cdot 132+1 \cdot 202+3 \cdot 102+1 \cdot 021+3 \cdot 022+1 \cdot 032+ \\
&+3 \cdot 011+4 \cdot 012+4 \cdot 001+4 \cdot 002+1 \cdot 312+1 \cdot 300+1 \cdot 101+1 \cdot 000+1 \cdot 100, \\
& p= 1 \cdot 122+1 \cdot 112+1 \cdot 102+1 \cdot 022+1 \cdot 032+1 \cdot 012+1 \cdot 002 .
\end{aligned}\right.
$$

The real solution would have the following form

$$
f(x, Q, p)^{t_{w_{w}}<100 t_{o}}=1 \cdot 022+1 \cdot 032+1 \cdot 012+1 \cdot 002=1 \cdot(0-2) .
$$

\section{Conclusions}

The article is about a procedure of combinatorial solving of weighting multi-valued sets of logical equations describing guidelines of designing in view of the morphological analysis with keeping RosserTurguette's postulates.

Weighting multi-valued set of logical equations describing guidelines of designing can be minimized separately or together with keeping the logical equivalence. In this way, we can also keep individual properties of each function.

It has been proved that in a general case, minimization of logical functions with weighting coefficients can be the same as without weighting coefficients. However, a better reflection of physical models of hydraulic sweep systems has been obtained in their mathematical models for example in overflow valves or proportional ones.

Increasing, decreasing or keeping such values unchanged in the readjusting process of the system to different work conditions can be coded in the multi-valued logic, whereas designing guidelines can be presented as a sum of multi-valued logical products. Solutions to the system of weighting multi-valued logical equations are versions of code changes in parameters $m, k$ and $d$ of all functions $x, Q$ and $p$ depending on time $t$. In the case of such assumptions, it is also possible to introduce conditions of uncertainty for appropriate logical products of designing guidelines, which means a partly described function in the automata theory. 


\section{Nomenclature}

$$
\begin{aligned}
d & - \text { the valve diameter }[\mathrm{m}] \\
d_{1} & - \text { inlet manifold diameter }[\mathrm{m}] \\
d_{2} & - \text { valve seat diameter }[\mathrm{m}] \\
\boldsymbol{F} & - \text { hydrodynamic reaction force }[\mathrm{N}] \\
K & - \text { spring constant, }[\mathrm{N} / \mathrm{m}] \\
\mathrm{m} & - \text { valve head mass }[\mathrm{kg}] \\
\left(m_{1}, \ldots, m_{n}\right) & - \text { multi-valued logical function of n variables }\left(\mathrm{m}_{1}, \ldots, \mathrm{m}_{n}\right) \text { - valued } \\
n & - \text { the number of different letters in the Boolean function, } \\
P & - \text { flow intensity }\left[\mathrm{m}^{3} / \mathrm{s}\right] \\
p_{\text {otw. }} & - \text { valve opening pressure }[\mathrm{MPa}] \\
p_{p} & - \text { pressure in the inlet part in a particular position }[\mathrm{MPa}] \\
p_{z} & - \text { pressure over the valve in a particular position }[\mathrm{MPa}] \\
Q_{1} & - \text { flow rate of the pumped liquid }\left[\mathrm{m}^{3} / \mathrm{s}\right] \\
Q_{2} & - \text { flow rate of the liquid coming out of the valve }\left[\mathrm{m}^{3} / \mathrm{s}\right] \\
Q_{m 1} & - \text { massive flow rate of the liquid going into the valve }\left[\mathrm{m}^{3} / \mathrm{s}\right] \\
Q_{m 2} & - \text { massive flow rate of the liquid coming out of the valve }\left[\mathrm{m}^{3} / \mathrm{s}\right] \\
S & - \text { spring bias force }[\mathrm{N}] \\
t & - \text { time }[\mathrm{s}] \\
V & - \text { the valve volume }\left[\mathrm{m}^{3}\right] \\
x & - \text { spring deflection }[\mathrm{m}] \\
x_{o} & - \text { free vibrations amplitude }[\mathrm{m}] \\
\left(x_{1}, x_{2}, x_{3}\right) & - \text { decision variables } \\
\beta_{p} & - \text { coefficient of compressibility }\left[\mathrm{m}^{3} / \mathrm{s}\right] \\
\eta & - \text { viscous friction coefficient }\left[\mathrm{Ns}_{\mathrm{s}} / \mathrm{m}\right] \\
\rho & - \text { liquid density }\left[\mathrm{kg} / \mathrm{m}^{3}\right] \\
&
\end{aligned}
$$

\section{References}

Deptuła A. (2014): Optimization of machine systems using logic equations and graph-structures. - DissertationDepartment of Mechanical Engineering, Technical University of Opole.

Deptuła A. (2011): Weighted logic equations design guidelines in discrete optimization of machine systems. - XL Conference on Applications of Mathematics, Zakopane 2011, Institute of mathematics PAN, Warsaw.

Deptuła A. and Partyka M.A. (2010): Application of game graphs in optimization of dynamic system structures. International Journal of Applied Mechanics and Engineering, vol.15, No.3, pp.647-656.

Deptuła A. and Partyka M.A. (2012): Solving weight multivalent logic equations describing the design guidelines in discrete optimization of machine systems. - XLI Conference on Applications of Mathematics, Zakopane 2010, Institute of Mathematics PAN, Warsaw 2010.

Francis J. and Betts P.L. (1997): Modelling incompressible flow in a pressure relief valve. proceedings of the institution of mechanical engineers. - Part E: Journal of Process Mechanical Engineering, vol.211, No.2, pp.83-93.

Giergiel J. (1990): Identification of mechanical systems. - Warsaw: PWN.

http://www.mathworks.com/products/simhydraulics.html

http://www.kirupa.com/developer/actionscript/depth_breadth_search.html.

Kurowski W. (2001): Modelling technical objects. - Manuscript Development, Płock. 
Partyka M.A. (1984): The Quine - Mc Cluskey minimization algorithm of individual multiple- valued partial functions for digital control systems. - 3rd Inter. Confer. Syst. Engin., Wright State University, Dayton.

Smith J. (2003): Parallel mechanisms. - Archive of Mechanisms, vol.2, No.24, pp.113-125, Warsaw.

Stępniewski M. (1994): Pomps. - Warsaw: WNT.

Suzuki K. and Urata E. (2003): Improvement of cavitation resistive property of a water hydraulic relief valve proc. The Eighth SICFP, 1, pp.265-276.

Żak J. and Stefanowski J. (1994): Determining maintenance activities of motor vehicles using rough sets approach.Proc. of Euromaintenance'94 Conference, Amsterdam.

Received: May 18, 2014

Revised: June 25, 2014 